\title{
Coates 图的两个消去定理
}

\author{
张 福 基 \\ (新期哈密沙范学校)
}

本文旨在证明 Coates 图的两个消去定理，正如文献 [1] 中所表明的那样，把图论技术用 到计算方法上是卓有成效的.

本文采用文献 [2] 中的一切符号与术语, 只是各有向边的重量不必一定是数, 可以是任意 环(如多项式)之元, 我们的讨论从推广文献 [2] 中定义 3.2 关于 1-因子的概念开始. 考察 1因子的各个分支（文献 [2] 中定义 1.10）易见 1-因子必由通过各顶点的互不相交的若干（有 向）圈构成,我们引入下述的

定义 1 图 $G$ 的一个顶点集合为 $I=\left\{i_{k} \mid k=1,2, \cdots, s\right\}$, 图 $G$ 中关于 $I$ 的部分 1-因 子指的是经过了 $I$ 中各顶点的若干互不相交的圈所构成的 $G$ 的子图且每一圈至少经过 $I$ 内的 一个顶点.

显然当 $I$ 与 $G$ 全部顶点集合一致时，此概念与 1 -因子一致。我们证明下述的

定理 $1 n$ 阶矩阵 $A$ Coates 图为 $G_{c}(A) . I=\left\{i_{k} \mid k=1,2, \cdots, s\right\}, s \leqslant n$ 为 $G_{c}(A)$ 的 顶点的子集, $h_{1}, h_{2}, \cdots, h_{m}$ 为 $G_{c}(A)$ 的关于 $I$ 的全部部分 1-因子, 则

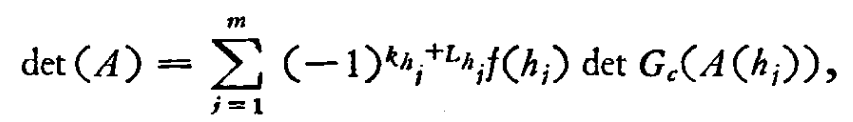

其中 $k_{h_{j}}$ 为 $h_{j}$ 的顶点数, $L_{h_{j}}$ 为 $h_{j}$ 所含不相交的圈数, $f\left(h_{j}\right)$ 为图 $h_{j}$ 的重量 (见文献 [2]p.140), $G_{c}\left(A\left(h_{i}\right)\right)$ 为图 $G_{c}(A)$ 中除去 $h_{j}$ 各顶点及与其相联的各边而得之图.

证 取 $G_{c}(A)$ 之任一 1-因子当中的至少含有 $i_{1}, i_{2}, \cdots, i_{s}$ 之一的各圈, 易见构成的子 图为 $h_{1}, h_{2}, \cdots, h_{m}$ 之一. 由此可将 $G_{c}(A)$ 的 1-因子分为集合 $Q_{1}, Q_{2}, \cdots, Q_{m}$, 其中 $Q_{i}$ 是含 有 $h_{i}$ 为子图的 1-因子的集合, 易见各 $Q_{i}$ 为互不相交. 事实上, 若 $Q_{i}$ 与 $Q_{i}$ 相交, 则交集中的 1-因子含有二互异部分 1-因子 $h_{i}$ 与 $h_{i}$, 因之至少有其中的一个顶点经过它有两个不同的圈, 此与 1-因子之定义矛盾.

今考虑 $Q_{i}$ 的重量，由于任一 $\omega \in Q_{i}$ 必含有一子图 $h_{i}$ 而在 $\omega$ 中除去 $h_{j}$ 后所得的子图即为 $G_{c}\left(A\left(h_{j}\right)\right)$ 之一 1-因子, 反之 $G_{c}\left(A\left(h_{i}\right)\right)$ 的任一 1-因子加上图 $h_{i}$ 后必构成 $G_{c}(A)$ 中之一 1因子 $\omega \in \Omega_{i}$, 故 $\Omega_{i}$ 中各 1-因子与 $G_{c}\left(A\left(h_{i}\right)\right)$ 中各 1-因子形成 1-1 对应. 二对应元重量的差 别为 $f\left(h_{i}\right) . G_{c}(A)$ 中某一 1-因子 $\omega$ 在文献 [2] p.144 定理 3.1 求和号中出现时，其符号为 $(-1)^{n+i}$ 而 $i$ 为 $\omega$ 所含互不相交之圈数. $G_{c}\left(A\left(h_{i}\right)\right)$ 的任一 1-因子在上述定理求和号中出 现时其符号为 $(-1)^{n-k_{h_{i}}+i-L_{h_{i}}}$. 二者符号差别为 $(-1)^{k_{h_{i}}+L_{h_{i}}}$, 故利用文献[2]定理 3.1, 定理 1 得证.

我们还引人下述的

本文 1977 年 12 月 13 日收到。修改稿 1979 年 3 月 6 日收到. 
定义 2 图 $G$ 的一个有向边的集合为 $I=\left\{e_{k} \mid k=1,2, \cdots, s\right\}$. 图 $G$ 的关于 $I$ 的部分 1-因子指的是经过 $I$ 中各边的若干互不相交的圈所构成的 $G$ 的子图，且每一圈中至少有 $I$ 中 的一个边。

显然当 $I$ 与 $G$ 全部边集合一致时,此概念与 1-因子一致, 我们可证明下述的

定理 2 阶矩阵 $A$ 的 Coates 图为 $G_{c}(A), I=\left\{e_{k} \mid k=1,2, \cdots, s\right\}$ 为 $G_{c}(A)$ 的各 个有向边的一个子集, $\mathfrak{C}=\left\{J_{l} \mid l=1,2, \cdots, 2^{s} J_{l} \subset I\right\}$ 为 $I$ 的一切子集的集合, 则

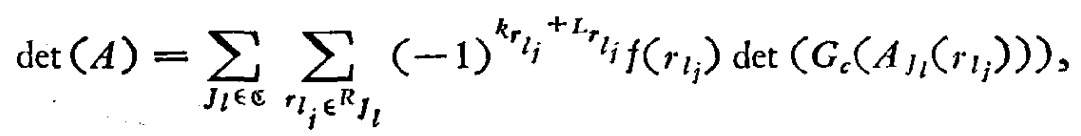

其中 $A_{J_{l}}$ 是 $G_{c}(A)$ 中除去 $I-J_{l}$ 中各边(但不除去其顶点)而得之图. $R_{J_{l}}$ 是图 $A_{l}$ 的关于 $J_{l}$ 的部分 1-因子的集合, 此类部分 1-因子记为 $r_{l_{j}}, A_{J_{l}}\left(r_{l_{j}}\right)$ 是 $A_{J_{l}}$ 中除去 $r_{l_{i}}$ 各顶点及与之相联 的各边而得之图, $f\left(r_{l_{j}}\right)$ 为 $r_{l_{j}}$ 的重量.

证 将图 $G_{c}(A)$ 的一切 1-因子分解为 $2^{s}$ 个子集 $\Omega_{l}, l=1,2, \cdots, 2^{s}, Q_{l}$ 是含有 $J_{l}$ 中 各有向边而不含有 $I-J_{l}$ 中各有向边的 1-因子的集合. 显然它们是互不相交的. 今对 $Q_{l}$ 再 行分解. 将 $Q_{l}$ 中的任一 1-因子的含有 $J_{l}$ 中某些有问边的圈放在一起, 显然是 $G$ 关于 $J_{l}$ 的一 个部分 1-因子. 今将含有部分 1-因子 $r_{l_{j}} \in R_{J_{l}}$ 的 $\Omega_{l}$ 中的 1-因子集合记为 $\Omega_{l_{j}}(j=1,2, \cdots$, $\left.m_{l}\right)$ ，又各 $Q_{l_{j}}$ 互不相交，否则它们公共元通过某一顶点有二不同圈与 1-因子的定义矛盾. 仿 定理 1 考虑重量及符号即得本定理.

当上述两定理中 $I$ 只含一顶点或一些边时即得下述的

推论 $1 n$ 阶方阵 $A$ 的 Coates 图为 $G_{c}(A), i$ 为其一顶点, $h_{1}, h_{2}, \cdots, h_{m}$ 为过 $i$ 的全部 圈, 则

$$
\operatorname{det}(A)=\sum_{j=1}^{m}(-1)^{k_{h_{i}}+1} f\left(h_{j}\right) \operatorname{det} G_{c}\left(A\left(h_{j}\right)\right) ，
$$

其中 $k_{h_{i}}$ 为圈 $h_{j}$ 的顶点数, $f\left(h_{j}\right)$ 为圈 $h_{j}$ 的重量, $G_{c}\left(A\left(h_{i}\right)\right)$ 为图 $G_{c}(A)$ 中除去 $h_{j}$ 各顶点及与 之相连的各边而得之子图.

推论 $2 n$ 阶方阵 $A$ 的 Coates 图为 $G_{c}(A),\left\{e_{i}\right\}=E$ 为 $G_{c}(A)$ 过顶点 $p$ 的一组有向 边, $r_{1}, r_{2}, \cdots, r_{m}$ 为含 $\left\{e_{i}\right\}$ 中边的全部圈, 则

$$
\operatorname{det}(A)=\sum_{i=1}^{m}(-1)^{k_{r}+1} f\left(r_{i}\right) \operatorname{det}\left(G_{c}\left(A\left(r_{i}\right)\right)\right)+\operatorname{det}\left(G_{c}(A(E))\right),
$$

其中 $k_{r_{j}}$ 为圈 $r_{i}$ 所含的顶点数, $G_{c}(A(E))$ 为图 $G_{c}(A)$ 中除去 $E$ 中各边 (但不除去其顶点) 所 得之图, $G_{c}\left(A\left(r_{i}\right)\right)$ 为图 $G_{c}(A)$ 中除去 $r_{i}$ 各顶点及与这些顶点相连的各边所得之图, $f\left(r_{i}\right)$ 为 图 $r_{i}$ 的重量.

证 应用定理 2 并注意到每一部分 1-因子只能由唯一的一个圈构成 ( $E$ 中各边均过顶 点 $p$ ). 事实上, 只有 $J_{l}$ 中含有一边且此边可构成圈或 $J_{l}$ 中含二边而二边在同一圈中出现时 $J_{l}$ 的部分 1-因子集合为非空.

从 Coates 图角度来证明此类定理系由文献 [3] 首先采用,文中讨论了有向图与无向图间 的关系 (用于本征网络). 从那里的方法可以得到与定理 $1 、 2$ 平行的无向图的结果, 事实上那 相当于对称方阵的情形,在此不鳌述. 此处指出, 处理本征网络时, 推论 1 与推论 2 的 $E$ 只含 有一个边情形. 关于无向图的结果由文献 [3] 得到由它可以推出两个早期结果 ${ }^{[4]}$ 又唐敖庆、江 元生 ${ }^{[5]}$ 用展开行列式的方法讨论了推论 $2, n=3$ 情形, 在他那里采用了完整偶图的概念. 事 
实上; 假定重量为 0 的边不存在, 具体计算将更方便 (可少考虑一些圈). 又推论 2 中 $E$ 只含 有一个边情形,则由刘为民 ${ }^{[6]}$ 首先证明,但采用本文不同的方法.

、本文提出的定理在不同领域内的应用,我们将专门讨论. 致谢: 对关臂直、张粪贤同志的帮助,作者仅致谢意。

\title{
参文款
}

[1] Tewarson, R. P., Sparse Matrices, Academic Press, New York and London, 1973.

[2] Chen, W. K., Applied Graph.Theory, North-Holland Company, Amsterdan and London, 1971.

[3] 张福基, 中国科学, 1979, 2: 154.

[4] Schwenk, A. J., Lecture Notes in Mathematics, No. 406, Springer, Berlin, 1974, 153.

[5] 唐敖庆、江元生, 科学通报, 22 (1977)，494.

[6]刘为民, 科学通报, 23 (1978), 65 .

\section{TWO THEOREMS OF ELEMINATION OF NODES AND EDGES FOR A COATES GRAPH}

\author{
Zhang Fu-ji (张福基)
}

Abstract

Let $I=\left\{i_{k} \mid k=1,2, \ldots, s\right\}$ be a set of nodes (edges) of graph $G$. We defined a 1-subfactor concerning $I$ as a set of disjoint directed circuits connected with all the nodes (edges) in $I$, every one in which is at least connected with (having) one node (edge) in 1 . Two theorems have been obtained.

Some special cases have been previously worked out by several authors. 